\title{
Síndrome hepatopulmonar en pediatría. Presentación de un caso y revisión de la literatura
}

\author{
Dr. Julio Maggiolo', Dra. Lilian Rubilar', Dra. Rebeca Paiva', \\ Dra. Gabriela Román², Dra. Carolina Campos³. \\ 1 Médico. Unidad Broncopulmonar. Hospital de Niños Dr. Exequiel González Cortés. \\ 2 Médico. Becada de Pediatría. Universidad de Chile. Hospital de Niños Dr. Exequiel González Cortés. \\ 3 Médico. Unidad de Cardiología. Hospital de Niños Dr. Exequiel González Cortés.
}

\section{HEPATOPULMONARY SYNDROME IN CHILDREN: A CASE REPORT AND LITERATURE REVIEW}

\begin{abstract}
Hepatopulmonary syndrome is a rare disease that affects patients of any age with acute or chronic liver disease. The prevalence and its outcome in children are unknown. Clinically are common shortness of breath, orthodeoxia and platypnoea, together with cyanosis, digital clubbing and spider naevi. Its diagnosis is based in a clinical triad characterized by liver disease with or whithout portal hypertension, hypoxemia and intrapulmonary vascular dilatation leading to right and left shunts, demonstrated by echocardiography with contrast, the perfusion lung scanning using technetium-labelled macro-aggregates albumin estimate the shunt fraction. Pulmonary angiography is useful to demonstrate macroscopic arteriovenous communications. The orthotopic liver transplantation is the only efficacy treatment in patients without several gas exchange abnormality.
\end{abstract}

Key word: Hepatopulmonary, liver disease, hypoxemia, pulmonary shunt, liver transplantation.

\section{RESUMEN}

\begin{abstract}
El síndrome hepatopulmonar es una enfermedad poco frecuente que afecta a pacientes de cualquier edad con enfermedad hepática aguda o crónica. La prevalencia y la evolución en pediatría son desconocidas. Clínicamente es común encontrar disnea de esfuerzo, platipnea y ortodeoxia, además de cianosis, acropaquia y nevi aracniformes. El diagnóstico está basado en una tríada clínica caracterizada por una enfermedad hepática con o sin hipertensión portal, hipoxemia y vasodilatación intrapulmonar que conduce a un shunt de derecha a izquierda, demostrado mediante ecocardiografía con contraste y cintigrafía de perfusión pulmonar con albúmina macroagregada marcada con tecnesio-99m que permite la estimación de la fracción del shunt. La angiografía pulmonar es útil para demostrar comunicaciones arteriovenosas macroscópicas. El trasplante hepático es el único tratamiento demostradamente eficaz, excepto en aquellos pacientes con un trastorno ventilatorio grave, por su mayor morbimortalidad.
\end{abstract}

Palabras clave: Hepatopulmonar, hepatopatía, hipoxemia, shunt pulmonar, trasplante hepático.

\section{INTRODUCCIÓN}

El síndrome hepatopulmonar (SHP) se caracteriza por una alteración en la oxigenación arterial debida a dilataciones intrapulmonares vasculares asociadas a disfunción hepática ${ }^{(1,2)}$. Entre las patologías hepáticas destacan la atresia de vías biliares, trombosis portal y otras causas de cirrosis. En un estudio de observó que el SHP aparece en un $8 \%$ de los niños con cirrosis o hipertensión portal grave $\mathrm{e}^{(2)}$.

El mecanismo etiopatogénico exacto de las dilataciones vasculares pulmonares es desconocido, la disminución del tono vascular se podría deber al desequilibrio entre agentes

Correspondencia: Dr. Julio Maggiolo M.

Teléfonos: 2042254-7893228.

E-mail:maggiolojulio@gmail.com

ISSN 07| 8-332। Derechos reservados. vasodilatadores y vasoconstrictores, los más estudiados son óxido nítrico (NO), monóxido de carbono (CO), endotelina (ET) y factor de necrosis tumoral alfa (FNT $\alpha)^{(1-3)}$.

Desde el punto de vista clínico se manifiesta con disnea, platipnea, ortodesoxia, acropaquias y cianosis labial. La ecocardiografía con contraste (suero salino) y la cintigrafía con macroagregados de albúmina marcada con tecnesio, permiten ver el paso del contraste a nivel sistémico, lo que evidencia la presencia de un cortocircuito de derecha a izquierda. La angiografía pulmonar distingue 2 patrones de comunicaciones arteriovenosas, a saber el tipo I o difuso y el 2 o focal, este último es el menos frecuente ${ }^{(4-6)}$. En el tipo 2 es posible la embolización. No existe ningún tratamiento farmacológico efectivo(5,6). El trasplante hepático resuelve esta patología en más del $80 \%$ de los casos, aunque puede tardar meses en desaparecer. El propósito de este artículo es describir a una paciente portadora de este síndrome, además de hacer una revisión de la literatura. 


\section{CASO CLÍNICO}

Niña 14 años de edad de procedencia rural, sin antecedentes perinatales ni respiratorios de importancia. Presenta disfunción familiar caracterizada por ser hija de madre soltera, bajo nivel socioeconómico y educacional, sin red de apoyo familiar. A los 5 años presenta hepatitis $A$, la biopsia hepática revela presencia de cirrosis con estudio etiológico negativo. Además presenta hipertensión arterial, hipercalciuria idiopática e hidronefrosis secundaria a litiasis pieloureteral derecha. La paciente concurre irregularmente a controles en gastroenterología y nefrología. A los 8 años se practica una nueva biopsia que revela hepatitis crónica con actividad portal-periportal grado 4, actividad lobulillar grado 2 y fibrosis grado 3, por lo que se diagnostica hepatitis crónica activa.

Evoluciona con hipertensión portal (HTP), presentando varios episodios de hemorragias digestiva secundarias a várices esofágicas II y várices gástricas que se manejan con propanolol. La paciente es derivada al Hospital Luis Calvo Mackenna para evaluar la posibilidad de practicársele trasplante hepático, sin embargo, la ecodopler abdominal demuestra imagen hipogénica noduliforme en segmento $V$ de $23 \times 16$ $\mathrm{mm}$ y afetoproteína elevada $(\mathrm{I} .260 \mathrm{ng} / \mathrm{ml})$ sugerente de hepatocarcinoma. Tomografía computada (TC) abdomen revela cirrosis multinodular, HTP y esplenomegalia, en tanto la resonancia nuclear magnética (RNM) demuestra daño hepático crónico avanzado, HTP, esplenomegalia y nódulo en el segmento $\mathrm{V}$.

A los 14 años de edad es derivada a la unidad de neumología, constatándose cianosis, taquipnea, platipnea, ortodeoxia e hipocratismo digital, saturación $\mathrm{O}_{2}\left(\mathrm{Sat}_{2} \mathrm{O}_{2}\right) 88 \%$ con aire, Radiografía $(\mathrm{Rx})$ tórax normal, TC tốrax muestra dilataciones vasculares, se decide hospitalizar para completar estudio. Se plantea el diagnóstico síndrome hepatopulmonar (SHP) por la presencia de la tríada: enfermedad hepática, hipoxemia y posible shunt intrapulmonar. Reunión multidisciplinaria se decide confirmar y evaluar la severidad del SHP, se solicitan gases arteriales (GSA) basales, test de hiperoxia, diferencia $\mathrm{Aa}$ (alvéolo-arterial) de $\mathrm{O}_{2}$, evaluar shunt mediante ecocardiograma contraste (burbuja) y cintigrama de perfusión pulmonar y apoyo con $\mathrm{O}_{2}$ ambulatorio.

\section{Tabla I. Patologías hepáticas asociadas a síndrome hepatopulmonar}

- Atresia de vías biliares: 9-20\%

- Cirrosis: 2-4\%

- Cirrosis e hipertensión portal grave: 8\%

- Trombosis portal: 0,5\%

- Hepatitis crónica activa

- Déficit a I antitripsina

- Enfermedad Wilson

- Tirosinemia

- Hepatitis aguda fulminante

- Rechazo agudo de trasplante hepático
Los exámenes de laboratorio revelan: hipoxemia $\mathrm{pO}_{2}$ 20,2 mmHg), Sat $\mathrm{O}_{2} 80 \%, \mathrm{HCO}_{3} 23$, diferencia Aa de $\mathrm{O}_{2}$ de 604 (valor normal < 15), test hiperoxia menor 300 $\mathrm{mmHg}$. Ecocardiograma con microburbujas de solución fisiológica (test de la burbuja), se visualizan burbujas en aurícula izquierda en el cuarto ciclo cardíaco. Cintigrama perfusión pulmonar con macroalbúmina marcada con Tc99, demuestra gran cortocircuito derecha a izquierda de 55\%, con captación de radiofármaco en cerebro y riñones. Al demostrarse shunt intrapulmonar se hace el diagnóstico de SHP, además se califica de severo por hipoxemia $\left(\mathrm{pO}_{2}<50 \mathrm{mmHg}\right)$, test hiperoxia con ascenso de la $\mathrm{pO}_{2}$ menor de $300 \mathrm{~mm} \mathrm{Hg}$, shunt significativo e HTP. Por existir fuerte sospecha de hepatocarcinoma, lo que asociado a las malas condiciones clínicas y socioeconómicas de la paciente no permitieron realizar trasplante hepático y la paciente fallece.

\section{DISCUSIÓN}

La asociación entre daño hepático y alteraciones en la función pulmonar fue descrita inicialmente por Fluckieger ( 1884) y posteriormente Kennedy y Knudson (1977) acuñaron el término síndrome hepatopulmonar (SHP) ${ }^{(1,2)}$. El SHP es una entidad clínica caracterizada por la tríada: oxigenación deficiente, shunt intrapulmonar, disfunción hepática, sin enfermedad cardiopulmonar intrínseca ${ }^{(1-3)}$. La oxigenación arterial deficiente está definida por hipoxemia $\left(\mathrm{PaO}_{2}\right.$ menor $\left.70 \mathrm{mmHg}\right)$, aumento de la diferencia $\mathrm{Aa} \mathrm{O}_{2}$, mayor $15 \mathrm{mmHg}$ (signo precoz) y test hiperoxia menor $300 \mathrm{mmHg} g^{(5,6)}$. La hepatopatía puede ser aguda o crónica, con o sin hipertensión portal, las más importantes se muestran en la Tabla I.

Desde el punto de vista de la etiopatogénico existe disminución del tono vascular pulmonar, debido a un desequilibrio entre factores vasodilatores y vasoconstrictores dentro de los que se cuentan: NO, CO, ET, FNT $\alpha$, VIP-Sustancia P, glucagón, prostaglandinas, estrógenos, ferritina, entre otros ${ }^{(5,6)}$. El $\mathrm{NO}$ es el agente vasodilatador más importante, se encuentra aumentado en el aire exhalado. Es así como el azul de metileno (inhibidor de NO) y L-NAME (inhibidor de NO sintetasa) mejoran transitoriamente la oxigenación. Además NO sintetasa presenta efecto angiogénico, lo que lleva a malformaciones AV pulmonares y cutáneas ${ }^{(7-9)}$.

Los eventos etiopatogénicos se pueden resumir como sigue, la endotelina I (ET- I) aumenta resistencia (R) vascular y al receptor endotelina B (ET-B) induce la NO sintetasa endotelial (eNOS), la que aumenta la producción de NO. Endotoxinas bacterianas y FNT $\alpha$ inducen a los macrófagos a aumentar la producción NO sintetasa inducible (iNOS), la que a su vez aumenta más la producción de $\mathrm{NO}$ y hemoxigenasa I ( $\mathrm{HO}$ I), que aumenta la producción de $\mathrm{CO}$ (Figura I). Todos estos eventos conducen a dilatación de los vasos pre y postcapilares, disminución de la respuesta vasoconstrictora pulmonar a la hipoxia, fístulas AV, circulación hiperdinámica en que la sangre venosa pasa rápidamente por los capilares pulmonares, lo que produce aumento del gasto cardíaco, mayor vasodilatación pulmonar y reducción de la difusión de $\mathrm{CO}$ (DLCO) lo que eleva los niveles de $\mathrm{COHb}$, todo lo cual lleva a la desoxigenación arterial(7-9). Clínicamente se caracteriza 


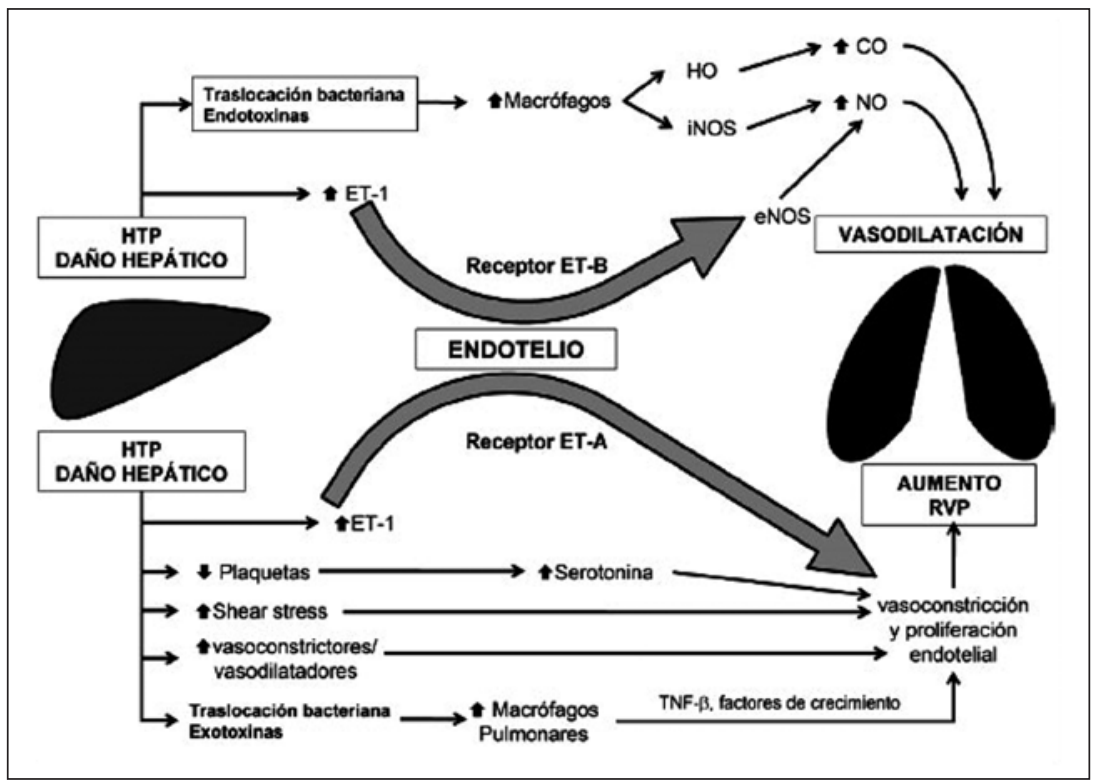

Figura I. Etiopatogenia del síndrome hepatopulmonar. Tomado de Zagolin M. Síndrome hepatopulmonar e hipertensión portopulmonar: dos entidades a diferenciar. Rev Chil Enf Respir 24: 29I-303. HO: hemoxigenasa; ET- I : endotelina I; CO: monóxido de carbono; iNOS: óxido nítrico sintetasa inducible; NO: óxido nítrico; eNOS: óxido nítrico sintetasa endotelial; HTP: hipertensión portal; ET-B: endotelina B. por disnea de esfuerzo pudiendo progresar hasta hacerse de reposo, Platipnea definida como mayor disnea sentado o en bipedestación, Ortodeoxia es el descenso de la $\mathrm{PaO}_{2}$ mayor de $4 \mathrm{mmHg}$ o más del 5\%, desde decúbito dorsal a bipedestación, hipocratismo digital o acropaquia, cianosis central y/o periférica y telangectasias aracniformes ${ }^{(7-9)}$.

En relación a la función pulmonar la espirometría y los volúmenes pulmonares son normales. Los gases arteriales son útiles para el diagnóstico y el pronóstico, característicamente se encuentra hipoxemia, sin retención $\mathrm{CO}_{2}$ y alcalosis respiratoria, debida a la hiperventilación que se observan en las hepatopatías, además hay aumento de la diferencia $\mathrm{AaO}_{2}$, la que es sensible y precoz, incremento $\mathrm{NO}$ exhalado, test hiperoxia positiva definido como ascenso menor de 300 $\mathrm{mmHg}$ de la oxemia, luego de la administración de $\mathrm{O}_{2}$ al $100 \%(7-9)$

Desde el punto de vista imagenológico la Rx tórax habitualmente es normal. Ecocardiograma con contraste de burbujas considerado como el patrón de oro para la detección de shunt intrapulmonar, consiste en agitar 10 cc de solución fisiológica, obteniéndose microburbujas (menores de 90 u) en vena periférica de extremidad superior, presenta gran sensibilidad y poca especificidad, es positiva en $40 \%$ cirrosis sin diferencia $\mathrm{AaO}_{2}$ elevada, ni hipoxemia. Normalmente las burbujas quedan atrapadas en la red vascular pulmonar, en el shunt pulmonar de derecha a izquierda aparecen en las cavidades izquierdas luego de más de 3-6 ciclos cardíacos, en cambio en el shunt intracardíaco se observan en menos de 3 ciclos (Figura 2) ${ }^{(7-9)}$.

Cintigrafía de perfusión pulmonar albúmina macroagregada marcada Tc-99 detección y cuantificación shunt intrapulmonar, normalmente los macroagregados de albúmina miden más de 20u quedando atrapados en la red vascular pulmonar, en el shunt intrapulmonar el radiofármaco es transportado y retenido en regiones extrapulmonares (más de 6\%), especialmente en cerebro, riñones, bazo, hígado, entre otros (Figura 3).

AngioTC/angiografía pulmonar son útiles en la detección de dilataciones arteriales, además se pueden demostrar fístulas AV, el patrón tipo I o fístulas difusas y el tipo II o fístulas focales, en estas últimas se debe considerar embolización vascular ${ }^{(7-9)}$. TC tórax es útil para descartar enfermedades respiratorias crónicas coexistentes (Figura 4). TC abdomen puede demostrar lesiones hepáticas cirróticas (nódulos), esplenomegalia, signos de HTP (Figura 5). Los criterios diagnósticos y de gravedad se resumen en la Tabla 2.

Se han utilizado diversos esquemas farmacológicos, sin resultados satisfactorios, como análogos de somatostatina, betabloqueadores, glucocorticoides, inmunosupresores, vasoconstrictores pulmonares, inhibidores de NO, antibióticos (norfloxacino), garlic, azul de metileno, preparados a base de

\section{Tabla 2. Criterios diagnósticos de síndrome hepatopulmonar}

- Presencia de hepatopatía

- Estadios tempranos sólo aumento de diferencia $\mathrm{Aa}_{\mathrm{PO}}$, luego hipoxemia

- Dif. $\mathrm{AaO}_{2}>15 \mathrm{mmHg}$ (normal $4-8 \mathrm{mmHg}$ )

- Criterios de severidad oxigenación anormal: leve, moderado, grave, muy grave*

- Test hiperoxia $(+)<300 \mathrm{mmHg}$

- Estadios avanzados todos los parámetros de desoxigenación alterados

- Ecocardiograma con contraste de burbujas: (+)

- Cintigrafía pulmonar: $(+)$ 

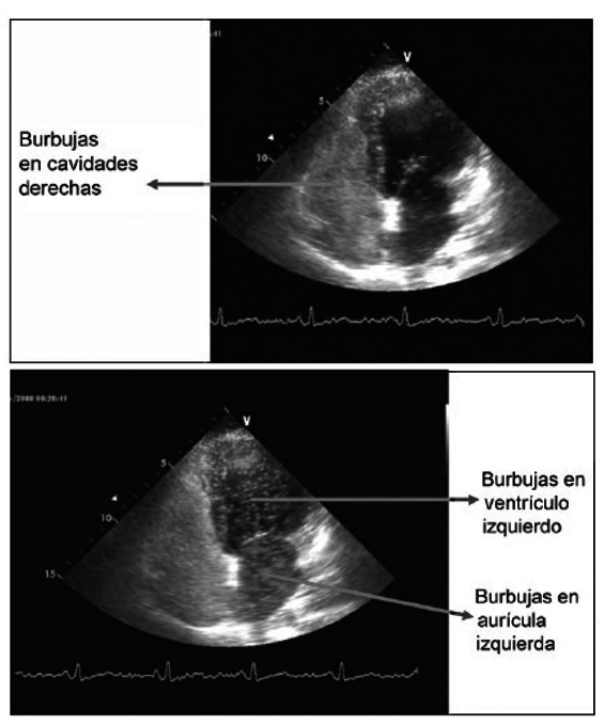

Figura 2. Ecocardiograma con microburbujas de solución fisiológica (test de la burbuja): las burbujas primero se observan en las cavidades derechas y luego en la aurícula izquierda, a partir del cuarto ciclo cardíaco. ajo $^{(10)}$. El tratamiento no farmacológico consiste en oxigenoterapia continua de bajo flujo a largo plazo, derivación portosistémica intrahepática transyugular, cavoplastía en Síndrome de Budd-Chiari, embolización con coils frente al patrón angiográfico tipo II, otros usados son agentes moduladores de ET-I, FNT $\alpha$ y HO-I.

El trasplante hepático es el único tratamiento efectivo, candidatos adecuados sólo representan el 10-20\%, idealmente con oxemia de 50-60 mmHg. Se observa resolución progresiva en alrededor de 85\%, meses a años postrasplante, las recidivas son excepcionales, deben ser donantes vivos. Idealmente la oxemia debería debería estar entre 60-80 $\mathrm{mmHg}$, con estricta vigilancia gasométrica, cuando la PaO2 es menor a $50 \mathrm{mmHg}$ la mortalidad postoperatoria es mayor a 30\%, si la selección es más estricta disminuye a 8,5\%, sin embargo, es discutible no trasplantar los casos severos ${ }^{(1)}$. El pronóstico sin trasplante es ominoso, siendo la sobrevida de I6-30\% a I año de hecho el diagnóstico, siendo peor si $\mathrm{PaO}_{2}$ es menor de $50 \mathrm{mmHg}$, shunt mayor de 20\%, HTP severa y si el test de hiperoxia es positivo(11-13).
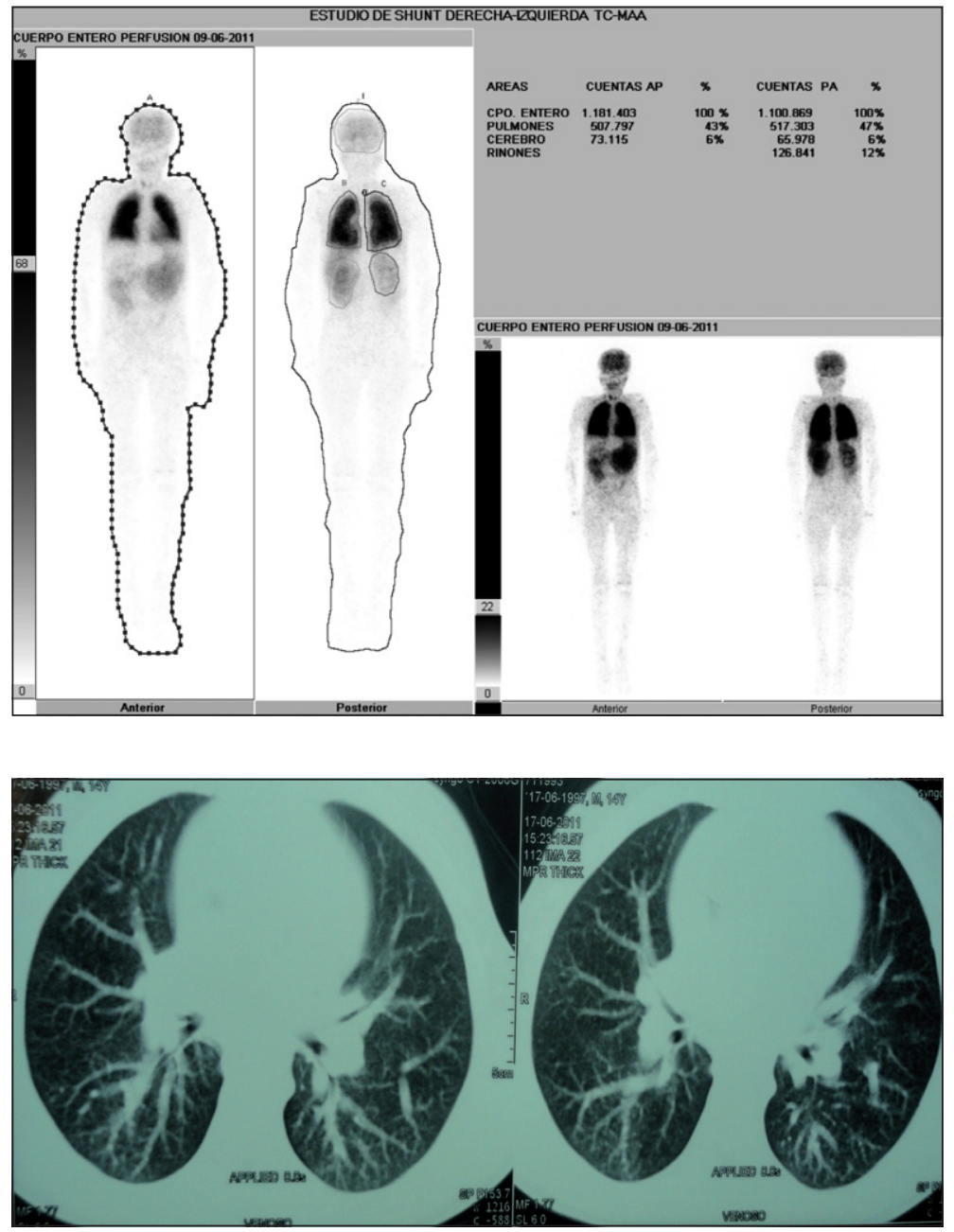

Figura 3. Cintigrama perfusión pulmonar con macroalbúmina marcada con Tc 99: gran cortocircuito derecha a izquierda de 55\%, con captación de radiofármaco en cerebro y riñones.

Figura 4. TC tórax: dilataciones vasculares. 


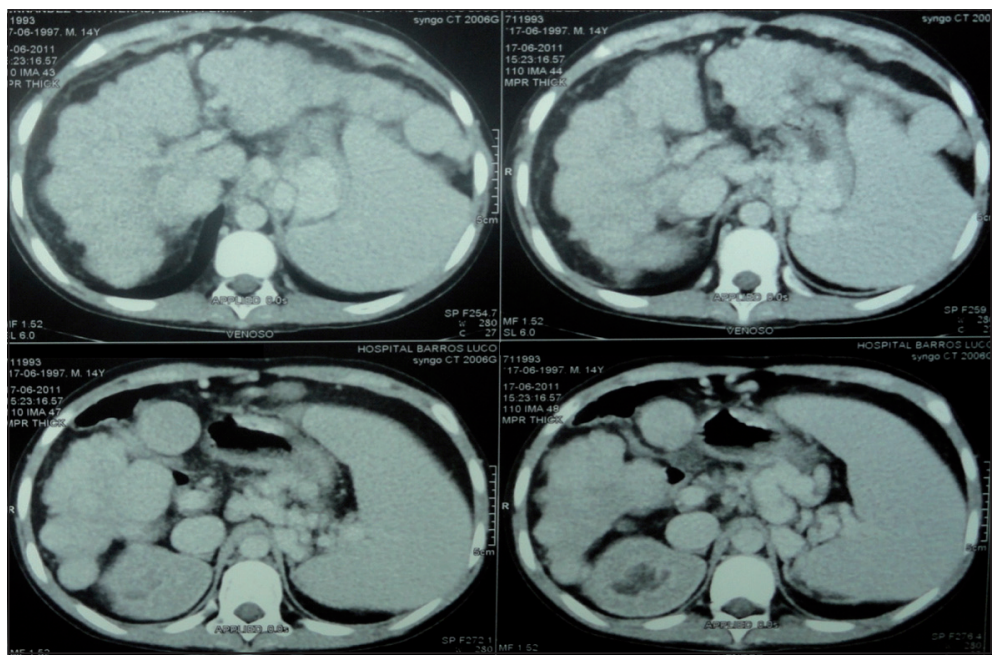

Figura 5. TC abdomen: cirrosis multinodular, HTP y esplenomegalia.

\section{REFERENCIAS}

I. Carrillo R. Síndrome hepatopulmonar (monografía). Rev Fac Med UNAM 200 I; 44: 207-II.

2. Palma D. The hepatopulmonary Syndrome. J Hepatol 2006; 45: 617-25.

3. Noli k. Prevalence of hepatopulmonary syndrome in children. Pediatrics 2008; 121 : e522-7.

4. Zagolín M. Síndrome hepatopulmonar e hipertensión portopulmonar: dos entidades a diferenciar. Rev Chil Enf Respir 2008; 24: 29|-303.

5. Kennedy TC. Exercise aggravated hypoxemia and orthodeoxia in cirrhosis. Chest 1977; 72: 305-9.

6. Muñoz S. Síndrome hepatopulmonar. Revista Hospital Clínico Universidad de Chile 2006; 17: 229-37.
7. Arguedas MR. Utility of pulse oximetry screening for hepatopulmonary syndrome. Clin Gastroenterol Hepatol 2007; 5: 749-54.

8. Rodríguez-Roisin R, Pulmonary-Hepatic Vascular Disorders. Eur Respir J 2004; 24: 861-80.

9. Rovira Amigo S. Síndrome hepatopulmonar en niños: evaluación y tratamiento. An Pediatr 2009; 71: 224-9.

10. Najafi M. Effect of oral garlic in children with hepatopulmonary syndrome. World J Gastroenterol 2006; I 2: 2427-3 I.

I I. Tumgor G. Childhood cirrhosis hepatopulmonary syndrome and liver transplantation. Pediatr Transplant 2008; 12: 353-7.

12. Willis AD. Hepatopulmonary syndrome in children is conventional liver transplantation always needed? Clin Transplant 2010 DOI: 10.1 | | |/j.1399-0012.2010.01378.x

13. Alonso Martínez JL. Síndrome hepatopulmonar: mayoría de edad clínica. Med Clin 2008; I30: 95-7. 